\title{
Knowledge of genetic testing for hereditary kidney cancer in Canada is lacking: The results of the Canadian national hereditary kidney cancer needs assessment survey
}

\author{
Philippe D. Violette, MD, FRCSC, ${ }^{*}$ Suzanne Kamel-Reid, MD, FRCSC; ${ }_{i}^{+}$Gail E. Graham, MD; ; \\ M. Neil Reaume, MD, FRCSC;: Michael A. Jewett, MD, FRCSC,; Melanie Care, MSc,; Joan Basiuk, RN,; \\ Stephen E. Pautler, MD FRCSC ${ }^{* a}$
}

\begin{abstract}
*Division of Urology, Department of Surgery, Schulich School of Medicine \& Dentistry, Western University, London, ON; ‘ Molecular Diagnostics, Department of Pathology, University Health Network, Toronto, ON; \$Eastern Ontario Regional Genetics Program, Children's Hospital of Eastern Ontario and the University of Ottawa, Ottawa, ON; ${ }^{2}$ Division of Medical Oncology, The Ottawa Hospital Cancer Centre and the University of Ottawa, Ottawa, ON; ${ }^{\circ}$ Department of Surgical Oncology, Princess Margaret Hospital, University Health Network, University of Toronto, Toronto, ON; £Department of Molecular Genetics, University Health Network, Toronto, ON; \#Kidney Cancer Research Network of Canada, Toronto, ON; 'Division of Surgical Oncology, Department of Oncology, Schulich School of Medicine \& Dentistry, Western University, London, ON
\end{abstract}

Cite as: Can Urol Assoc J 2014;8(11-12):e832-40. http://dx.doi.org/10.5489/cuaj.2415 Published online November 24, 2014.

\section{Abstract}

Introduction: Treatment of hereditary renal cell carcinoma (HRCC) requires a multidisciplinary approach that may involve medical oncologists, geneticists, genetic counsellors, and urologists. The objective of our survey was to obtain current and representative information about the use and perceived importance of genetic testing for HRCC in Canada.

Methods: A self-administered web-based survey was provided to Canadian medical oncologists, geneticists, genetic counsellors, and urologists in collaboration with their respective associations. The survey was created through an iterative process in consultation with the Kidney Cancer Research Network of Canada and contained both quantitative and qualitative components. The survey was designed to be exploratory and results were compared across regions.

Results: The overall response was low $(6.6 \%)$. Of the respondents, $42 \%, 33 \%$, 19\%, 5\% were genetic counsellors, urologists, medical oncologists and medical geneticists, respectively. Of the respondents, $62.7 \%$ described their practice as academic, and $37.3 \%$ described it as non-academic. Non-academic respondents tended to refer for genetic counselling less frequently than academic $(48.6 \%$ vs. $67.2 \%)$. Most respondents believed that genetic testing for HRCC was available (82.8\%), although $47.7 \%$ did not know which tests were available. This observation was consistent across provinces. Testing for Von Hippel-Lindau syndrome was given the highest priority among respondents. Limited provider knowledge, clinical guidelines, institutional funding, access, and poor coordination between disciplines were cited as barriers to testing.

Interpretation: There is a need to increase provider knowledge of genetic testing for HRCC. These findings support the development of practice guidelines and national strategies to improve coordination of specialists and access to genetics services. Limitations of the present study include low survey response which did not allow for inferential analysis by geographic region or respondent specialty.

\section{Introduction}

Renal cell carcinoma (RCC) is the sixth most common cancer in men and the eighth most common in women. ${ }^{1}$ In Canada there were 5900 new diagnoses and 1740 deaths from RCC in $2013 .^{2}$ Most are sporadic; however between $4 \%$ and $8 \%$ can be classified as hereditary. ${ }^{3,4}$ Some authors suggest that the true incidence of hereditary RCC (HRCC) may be much higher, but current estimates suffer from under diagnosis and underreporting. 5,6 Several hereditary syndromes have been identified, the most common of which are Von HippelLindau (VHL), hereditary papillary RCC (HPRCC), hereditary leiomyomatosis and RCC (HLRCC), Birt-Hogg-Dube syndrome (BHD), and tuberous sclerosis complex (TSC). Each of these syndromes has distinctive presentation, associated conditions, aggressiveness, causal genes, and penetrance.

Treatment of hereditary RCC requires a multidisciplinary approach that may involve medical oncologists, dermatologists, geneticists, genetic counsellors, and urologists. The ability to provide a timely diagnosis and effective treatment plans is dependent on the availability of appropriate genetic testing for our patients. Additionally, genetic testing can have clinical utility for the relatives of patients, especially for conditions where screening has demonstrated efficacy. ${ }^{7,8}$ However, the current state of genetic testing for HRCC across Canada is unknown. It is essential to know the perceived clinical needs for genetic testing, gaps in availability between jurisdictions to identify areas for future research efforts and resource allocation. ${ }^{8}$ Therefore, we conducted a 
national needs assessment survey to better understand the national and regional priorities for genetic testing of HRCC.

\section{Methods}

\section{Development and design of questionnaire}

The Kidney Cancer Research Network of Canada (KCRNC) evolved from 3 Canadian Kidney Cancer Forums held in 2008, 2009, and 2011. The KCRNC represents a pan-Canadian network connecting kidney cancer researchers, survivors, clinicians, and healthcare partners. In 2013, a Genetics Awareness and Practices working group was convened to generate the initial draft of topic priorities to be evaluated. The focus group was made up of 16 members and included urologists, medical oncologists, genetic counsellor, medical geneticists, laboratory scientists, and patient representatives. The key areas of interest identified were: demographic distributions of access, patterns of use, perception of availability, perceptions of barriers to access, and knowledge regarding indications and perceptions of usefulness of genetic tests for HRCC. From these key areas a questionnaire was drafted and further refined through an iterative feedback with group members, which resulted in the final 14-question survey encompassing 4 topic areas. The questions related to demographic information of respondents, availability and use of genetic testing for HRCC, knowledge of indications for use, perception of barriers and limitations to genetic testing for HRCC. Perceived barriers were assessed initially through qualitative analysis of elicited responses, and additionally through quantitative assessment of pre-defined potential barriers to genetic testing.

\section{Participants and conduct}

Prior to conducting the survey ethics approval was obtained from Western University Research Ethics Review Board (\#103891) and Lawson Health Research Institute (R13314). The survey was distributed in collaboration with Canadian Association of Medical Oncologists (CAMO), Canadian Association of Genitourinary Medical Oncologists (CAGMO), Canadian Dermatology Association (CDA), Canadian College of Medical Geneticists (CCMG), the Canadian Association of Genetic Counsellors (CAGC), and the Canadian Urological Association (CUA). Each participating association was requested to distribute an invitation directly to their membership by email every 2 weeks for 6 consecutive weeks. The CDA was unable to comply with this protocol, but included an invitation to participate in the survey embedded in their monthly newsletter. As a result there were no responses from the CDA members and as such they were excluded from the study. The self-administered survey was available in French and English and was conducted using an internet based platform ${ }^{9}$ from September 24, 2013 to December 13, 2013. The survey was designed and piloted to take 10 minutes. Post-survey analysis revealed that respondents took a mean of 11.28 minutes to complete the survey.

\section{Analysis}

The survey platform allowed for both closed- and openended question types. Closed-ended questions were described for the population overall using percentages for categorical answers and mean with standard deviation (SD) for continuous data. Fewer responses were obtained from Saskatchewan, Manitoba, New Brunswick, Prince Edward Island, and Newfoundland. Therefore responses were grouped into 4 regions: Western Canada (British Columbia, Alberta, Saskatchewan, Manitoba), Ontario, Quebec and Eastern Canada (Newfoundland, Prince Edward Island, Nova scotia, New Brunswick). Answer choices were then grouped by region and compared with univariable statistics using SPSS version 20 (IBM Corp., Armonk, NY). This study was designed to be exploratory and therefore inferential statistics were not performed. Open-ended qualitative questions were summarized using an adaptation of qualitative description analytic strategies as described by Neergaard and colleagues. ${ }^{10}$ Responses to open-ended questions were organized into responses with similar content. Raw themes were then extracted from each response. These raw themes were then grouped into common themes and subthemes, which captured the ideas expressed by the respondents. Additional synthesis and refinement were carried out to more clearly present qualitative findings.

\section{Results}

\section{Respondent characteristics}

The overall response was low, with 136 respondents of 2054 invited (6.6\% response rate). Healthcare providers whose primary location of practice was outside of Canada $(n=8)$ or who were not involved with patient care $(n=2)$ were excluded. Therefore, the total number of responses for analysis was 126 (6.1\% overall). However, the sampling strategy inadvertently included retired members $(n=124)$, non-specialist members $(n=101)$ and members who practice outside of Canada $(n=169)$. When we adjusted for the study inclusion criteria, the overall response rate was $7.6 \%$.

Of the respondents, $42 \%, 33 \%, 19 \%$, 5\% were genetic counsellors, urologists, medical oncologists and medical geneticists, respectively. The average number of years in practice was $10.6 \pm 8.6$. Geographically, $27 \%$ of respon- 
dents practiced in Western Canada, $43 \%$ in Ontario, $12 \%$ in Quebec and 18\% in Eastern Canada (Fig. 1). Representatives of each specialty responded from each geographical region. Among respondents, $37 \%$ identified their practice as primarily non-academic. Respondents were more likely to identify as primarily academic if they were from Western Canada $(64.7 \%$ vs. $35.3 \%)$, Ontario (63.0\% vs. $37.0 \%)$, or Quebec $(86.7 \%$ vs. $13.3 \%)$ in contrast with Eastern Canada (43.5\% vs. $56.5 \%)$. Most medical oncologists (83\%), medical geneticists $(83 \%)$, and urologists $(68 \%)$ who responded to the survey identified themselves as academic. A small majority $(54 \%)$ of genetic counsellors considered their primary practice as non-academic.

\section{Use of genetic testing and perceived availability}

We asked respondents a series of questions designed to elicit the actual use of genetic testing for HRCC across Canada. Overall, $62 \%$ of respondents referred for genetic testing. There was an increase in the referral rate in Ontario relative to the other regions in Canada (71\% vs. $51 \%, p<0.04$ (Fig. 2). Similarly, academic practitioners reported increased referral for genetic testing (Fig. 3) over non-academic practitioners.

The actual use of genetic testing may be reflective of the perception of availability of genetic testing for HRCC. Of respondents, $82.8 \%$ believed that genetic testing for HRCC was available in their regions of practice. However, $47.7 \%$ did not know which tests were available. This observation was consistent across jurisdictions (Fig. 4). VHL testing was

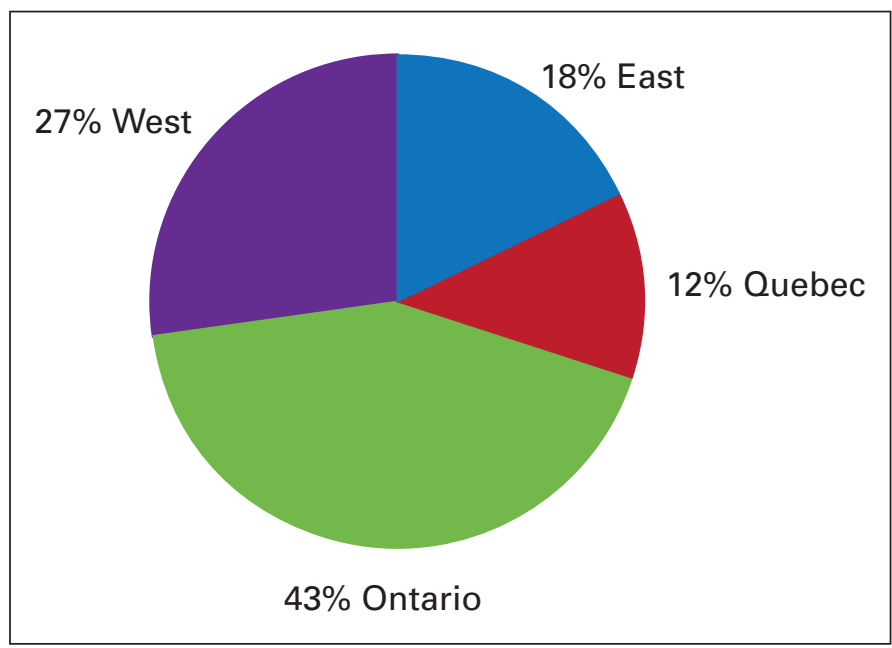

Fig. 1. Regional distribution of respondents.

most commonly perceived as available $(52.3 \%$ of respondents overall). Testing for other genes commonly associated with HRCC syndromes were generally thought to be unavailable (Met $37.5 \%$, FH 28.4\%, FLCN $22.7 \%$, TSC1 $31.8 \%$, TSC2 29.5\%).

When asked to rank a list of potentially available genetic tests in order of priority, respondents indicated VHL as a priority test ( $77.5 \%$ of respondents). All other genetic tests were not clearly identified as high priority (MET, FH, FLCN, TSC1, TSC2, SDHD, SDHC, SDHB, SDHA, PTEN).

Access to genetic testing was felt to be adequate by $58.8 \%$ of respondents. This observation was consistent

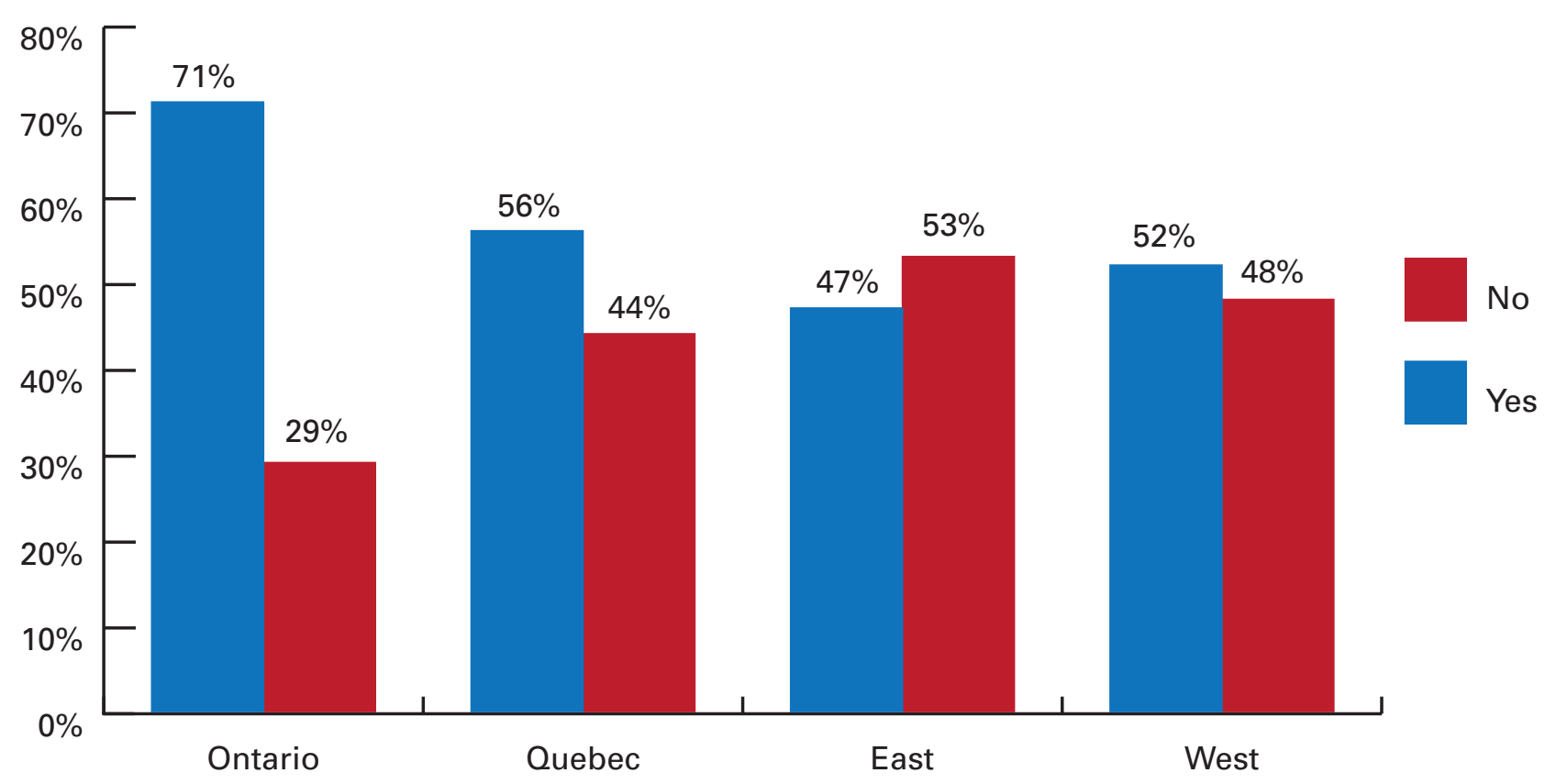

Fig. 2. Referral for genetic testing for hereditary renal cell carcinoma by region. 


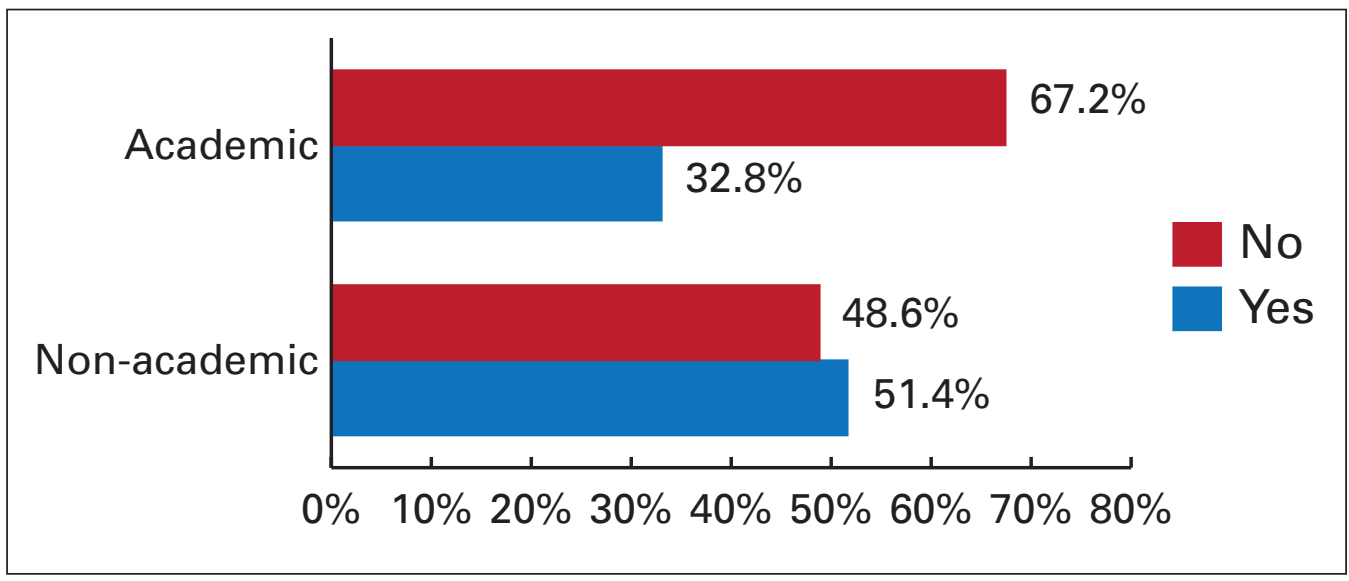

Fig. 3. Referral for genetic testing by academic versus non-academic practice setting.

across regions as follows: Ontario 56.8\%, Quebec 55.6\%, Eastern Canada 61.5\%, and Western Canada 61.5\%. Given a scenario in which genetic testing was freely available, practitioners reported a wide range in the frequency of use, with a mean of 7 tests per year (SD 8). The distribution of responses demonstrated a strong positive skew in which a small number of respondents reported that they would use tests up to 30 to 45 times per year.

Respondents were asked which healthcare practitioners should order genetic testing for hereditary kidney cancer syndromes. Most identified medical geneticists and genetic counsellors as practitioners who should order genetic tests (Fig. 5). A significant minority indicated that medical oncologists, urologists, and surgical oncologists should also be responsible for ordering genetic tests for HRCC. On open-ended questions, several respondents indicated that any practitioner who felt they had sufficient competence should order these tests.

\section{Knowledge of indications}

We asked respondents to select whether genetic testing should be ordered for a patient with a renal mass in 20 scenarios to assess knowledge base (Table 1). Thirteen of these scenarios were adapted directly from the CUA Consensus Guideline and as such were considered recognized indica-

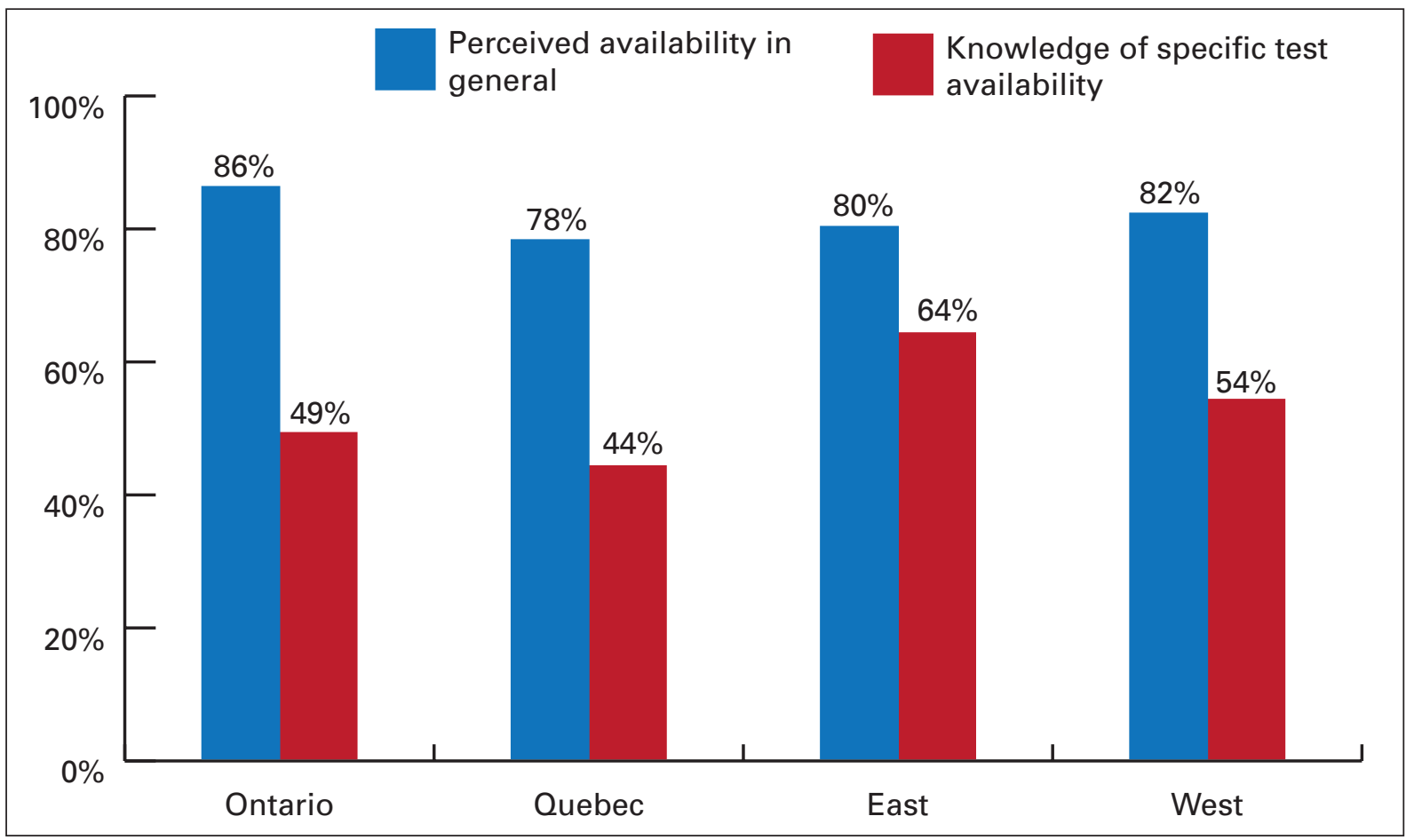

Fig. 4. Knowledge of availability of genetic tests for hereditary renal cell carcinoma. 
Violette et al.

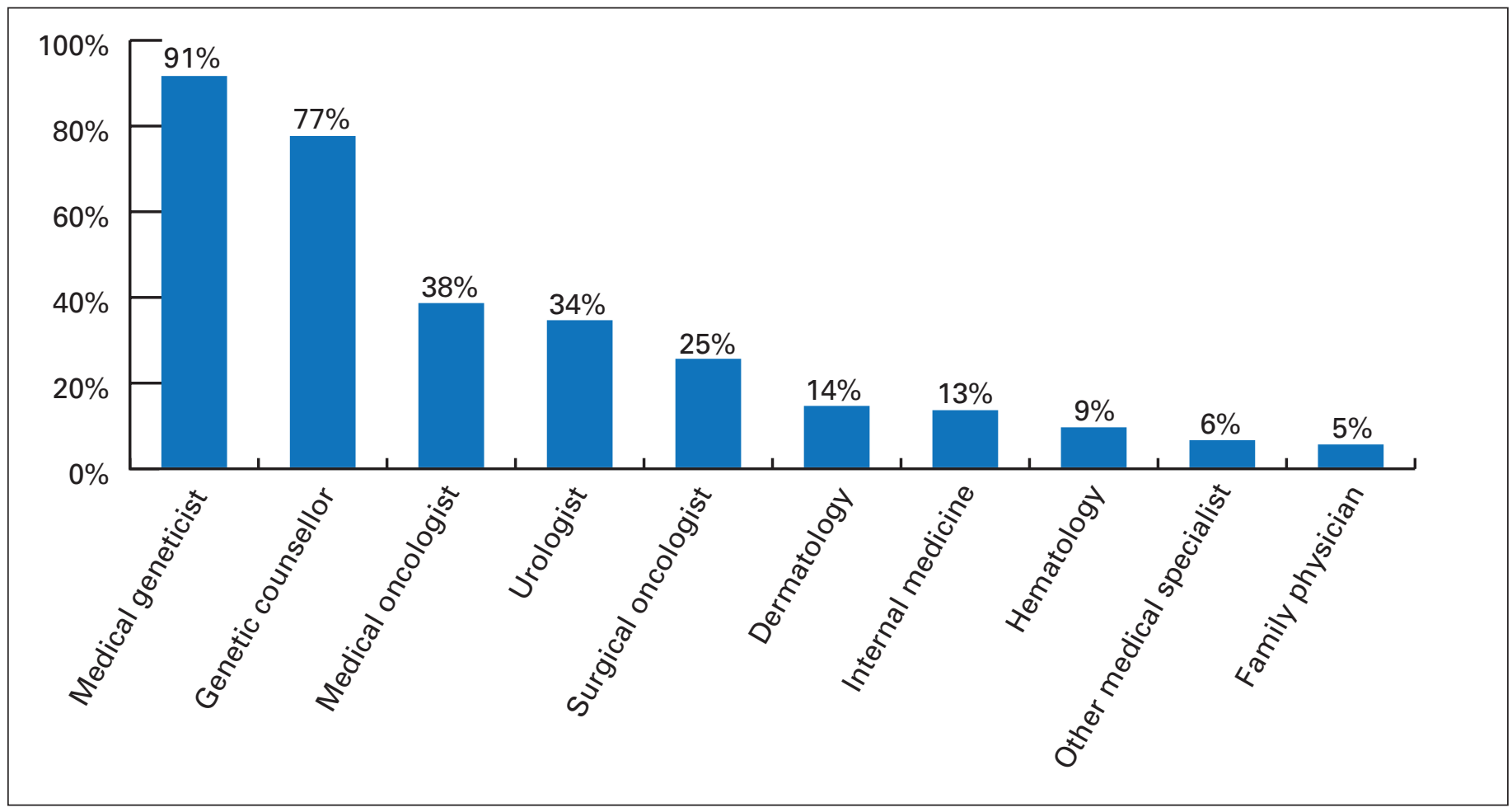

Fig. 5. Specialists healthcare practitioners felt should be responsible for ordering genetic tests for hereditary renal cell carcinoma.

tions for genetic testing for HRCC. ${ }^{11}$ Of these scenarios, a family history of a known HRCC-related syndrome was the most commonly recognized indication for genetic testing $(79.7 \%-87.3 \%)$. Surprisingly, a minority of respondents recognized a renal mass of unusual histology as an indication for genetic testing (34.2\%). Similarly, age $\leq 35$ years, bilateral or multifocal renal masses, and concomitant pheochromocytoma were not recognized as indications for genetic testing by many respondents (34.4-44.3\%). Additionally, a minority of respondents would order genetic testing for clinical situations in which genetic testing is not clearly recognized (Table 1).

\section{Limitations and barriers to genetic testing for HRCC}

Two open-ended questions were used to elicit the perceived limitations and barriers to the use of genetic testing for HRCC. From qualitative analysis of these questions, 3 common themes emerged: (1) resources, (2) knowledge, and (3) clinical practice and perception. Within each common theme, several subthemes characterized the barriers and limitations to the use of genetic testing for HRCC (Table 2).

The respondents were asked to assess the impact of potential barriers on a 5 point scale (0-none, 1 -little, 2-moderate, 3-high, 4-very high). A pre-defined list of 17 potential barriers was provided (Table 3). The top 5 barriers identified as either high or very high impact were provider knowledge about availability, provider knowledge about clinical impact, provider knowledge about HRCC, lack of clinical guidelines and long wait times for genetics clinic referral.

\section{Discussion}

\section{Availability and use}

Our survey demonstrated that most respondents believed genetic testing was available in their jurisdiction (82.8\%); however, a large proportion (41.2\%) believed that patient access remained inadequate. This is similar to attitudes expressed in a survey of cancer physicians in Ontario likely to order or use molecular oncology testing conducted by Miller and colleagues in 2006. ${ }^{12}$ In their survey, only 54\% of physicians agreed that patients had access to molecular oncology testing according to standard of care. This difference may reflect increasing awareness among users of genetic testing over the past 8 years. However, our survey also suggests that provider awareness is limited to a general belief in access, but does not translate to knowledge of availability of specific tests. Among respondents, $47.7 \%$ reported not knowing which specific genetic tests were available in their region. This represents a clear limitation to patient access. We speculate that this is a reflection of limited regional guidelines, heterogeneous procedures to gain approval for 


\section{Table 1. Clinical situations in which respondents would} order genetic testing for HRCC

Clinical characteristic of patient with a renal mass $\begin{gathered}\text { Percent who } \\ \text { would order } \\ \text { genetic testing }\end{gathered}$

\section{Recognized indication as per CUA Consensus} Guideline

Patient reports a family member with a confirmed diagnosis of $\mathrm{VHL}$

$87.3 \%$

Patient reports a family member with a confirmed diagnosis of familial papillary RCC

Patient reports a family member with a confirmed diagnosis of BHD

Patient reports a family member with a confirmed diagnosis of $\mathrm{HL}$ and RCC

Patient reports a family member with a confirmed diagnosis of TSC

Concomitant skin leiomyomas, skin

fibrofolliculomas, multiple skin papules

(excluding basal cell cancers, seborrheic

keratoses or melanomas)

Hemangioblastoma of the retina, brainstem,

cerebellum, or spinal cord

Bilateral renal masses

Multifocal renal masses

Pheochromocytoma

Patient with first- or second-degree relative with

a renal mass

History of pneumothorax

Unusual histology (e.g., oncocytic, hybrid etc...)

Age-related indication

Age $\leq 35$ years

Age between 35 and 65 years

Age over 65 years

Not a recognized indication for genetic testing

History of first-degree relative with

pneumothorax

History of first-degree relative with

pheochromocytoma

Solitary renal mass

Metastatic lesion with known primary RCC

HRCC: hereditary renal cell carcinoma; CUA: Canadian Urological Association; VHL: Von

Hippel-Lindau syndrome; RCC: renal cell carcinoma; BHD: Birt-Hogg-Dubé syndrome;

HL: hereditary leiomyomatosis; TSC: tuberous sclerosis complex.

selective genetic testing, and a rapidly changing landscape for genetic testing across jurisdictions in Canada.

The impact of uncertainty on delivery of healthcare is reflected in the heterogeneity of provincial regulations ${ }^{13}$ and the use of genetic testing across regions. Adair and colleagues conducted a qualitative description of Canadian genetic healthcare providers from British Columbia, Alberta, Manitoba, Ontario, Quebec, and Nova Scotia. ${ }^{14}$ They concluded that decision-making with regard to genetic testing in Canada was largely ad hoc and dependent on local priorities with regard to resource allocation. This observation
Table 2. Barriers to genetic testing

\begin{tabular}{ll}
\hline Common Themes & \multicolumn{1}{c}{ Subthemes } \\
\hline Resources & Lack of funding \\
& Access to Genetics Services \\
& Cost of testing \\
Knowledge & About HRCC \\
& Availability of tests \\
& Treatment options \\
& Benefits of testing \\
& Formal guidelines \\
Clinical practice and perception & Out of province/country testing \\
& Lack of coordination between \\
& specialists \\
& Need for more \\
& subspecialisation \\
& Rare not important \\
& Concern of discrimination \\
& Genetic testing as research only \\
\hline
\end{tabular}

Table 3. Proportion of respondents who assigned high or very high importance to the potential barrier

$\begin{array}{ll}\text { Potential barrier to the use of genetic } & \begin{array}{c}\text { Ranked high or very } \\ \text { high importance }\end{array}\end{array}$

Limited provider knowledge about

availability of genetic testing in their area

$67.2 \%$

Limited provider knowledge about the

clinical impact of genetic testing

$64.8 \%$

Limited provider knowledge about

hereditary renal cancer syndromes

$63.5 \%$

Lack of clinical guidelines

$55.3 \%$

Lack of evidence-based clinical information

to support utility of testing

$46.5 \%$

Barriers to Genetics clinic referral (such as long wait times)

$41.3 \%$

Test results will not affect treatment $\quad 22.6 \%$

Cost of tests is prohibitive $\quad 20.3 \%$

Lack of time, resources to educate patients $\quad 20.3 \%$

Potential impact on patient or family

members (inability to get life insurance,

$20.3 \%$

psychosocial implications)

Insufficient regulatory framework $\quad 18.9 \%$

Test results take too long $\quad 17.3 \%$

Too much paperwork/bureaucracy $\quad 17.3 \%$

Approval process takes too long $\quad 14.9 \%$

Lack of reimbursement for following up $\quad 14.1 \%$

genetic tests

$13.4 \%$

Patient anxiety regarding test results

$9.5 \%$

was also reflected in our survey, in which the use of genetic testing for HRCC was considerably greater in Ontario than other regions ( $71 \%$ vs. $51 \%$ ). We suggest that the increase in use of genetic testing in Ontario is a result of efforts to increase awareness and coordinate resources. For exam- 
Violette et al.

ple, in 2009 the Ontario Genetics Secretariat distributed a white paper publication for senior hospital leaders and Local Health Integration Networks (LHIN)..$^{15}$ Likewise, publically accessible resources, such as Genetic Resources Ontario, ${ }^{16}$ have been created to inform patients and family members. Consequently, this suggests that efforts to develop national treatment guidelines and funding strategies are warranted.

Technological advances in gene sequencing, with relative facility and decreased cost of next generation-sequencing are changing the landscape of genetic testing in Canada. ${ }^{17}$ This increased availability combined with increased patient expectations of access ${ }^{18}$ will result in an exponential increase in the demand for genetic testing in Canada. This poses a potential dilemma for providers and policy makers. Limited budgetary constraints and the number of trained specialist providers will not be adequate to cope with the demand for genetic testing. This tension may jeopardize patient care if an adequate model of healthcare delivery is not developed.

The management of HRCC can provide an ideal context in which to model systems of healthcare delivery in the age of personalized medicine. HRCC is diagnosable, with a limited number of currently available genetic tests and screening guidelines in Canada. As HRCC is less prevalent than other forms of cancer, the costs incurred for genetic testing are more manageable in a publically funded healthcare system. In this way healthcare delivery can be tailored to specific Canadian jurisdictions with limited financial impact. Lessons learned from devising equitable systems of care for HRCC can subsequently be extrapolated to more prevalent cancers and may represent significant cost-savings in the long term.

\section{Gaps in knowledge}

A recent Canadian consensus guideline has elaborated the indications for genetic testing. ${ }^{11}$ The consensus was developed from a multidisciplinary group of medical geneticists, genetic counsellors, medical oncologists, and urologists and was intended to reflect the common practice of specialist practitioners in these areas. By design, our survey attempted to capture the practice patterns from a broader sampling of these same disciplines. Our results demonstrate an important gap between recommended practice and actual knowledge of the indications for genetic testing in HRCC. Furthermore, on analysis of qualitative questions, one of the most frequently sited barriers to the use of genetic testing for HRCC was the absence of recognized guidelines. This supports the need for efforts to further disseminate current guidelines and the need to develop additional guidelines focussing on algorithms for diagnosis and treatment of suspected HRCC. Similarly, formal knowledge translation activities should be integrated prospectively into guideline development.
Our survey revealed a significant gap in provider knowledge of indications for evaluation of HRCC. Young age of diagnosis is a well-established risk factor for HRCC, although there is no consensus age cut-off in the literature. ${ }^{19-23}$ Shuch and colleagues recently evaluated the age distribution of RCC from the SEER-17 (Surveillance, Epidemiology, and End Results) registry and the Hereditary Kidney cancer registry of the National Cancer Institute to define a specific age threshold for genetic testing. ${ }^{6}$ The authors found the highest sensitivity and specificity corresponded to an age threshold of $\leq 46$ years. The CUA consensus guideline cut-off of $\leq 45$ years is in accord with these findings. ${ }^{11}$ To gather a broader consensus from Canadian practitioners, our survey divided age into 3 categories ( $\leq 35,35-65,>65$ years). Our hypothesis was that the younger age category would be most clearly identified as an indication for testing. Surprisingly, 34\% of respondents did not consider age $\leq 35$ years an indication for testing, while $54 \%$ would still consider genetic testing in patients over 65 . Similarly, other accepted indications for genetic testing include patients with tumours that are bilateral, multifocal, or of unusual histology. In contrast to our expectation, only $59 \%, 58 \%$, and $35 \%$ of respondents indicated that they would order genetic testing in these circumstances, respectively. This result indicates a significant discrepancy between the Canadian Consensus Guideline and common practice. As such, this reveals the need for greater efforts toward provider education and dissemination of guidelines.

With many advances in personalized medicine on the horizon, one can expect the knowledge of the genetic contribution to many known cancers to increase exponentially. Some authors suggest that up to $60 \%$ of patients diagnosed with sporadic RCC have a hereditary predisposition. ${ }^{4}$ Likewise the complexity of tumour heterogeneity among RCC is becoming increasingly understood. ${ }^{24-26}$ Although this heterogeneity poses a challenge to biomarker development, tailored therapies for somatic RCC are increasingly dependent on underlying genetic makeup of the tumours involved. ${ }^{27,28}$ Therefore it is critical to adequately support screening programs for HRCC; efforts to optimize care for these patients play an important role in advancing our understanding of the larger population with sporadic RCC. ${ }^{24}$

\section{Perceived barriers}

Barriers to genetic testing were assessed using both qualitative and quantitative methods in our survey. Qualitatively, 3 common themes emerged from open-ended questions: (1) resources, (2) knowledge, and (3) clinical practice and perception (Table 2). Quantitatively, the most commonly identified barriers to the use of genetic testing were provider knowledge about availability (67\%), clinical impact (65\%), HRCC 
(64\%), lack of guidelines (55\%), and wait times for genetic clinic referral $(41 \%)$. To our knowledge no other survey has been conducted to identify the barriers to the use of genetic testing specifically for patients with HRCC. Research regarding barriers to genetic testing and personalized medicine in other contexts has drawn similar conclusions.

Limited resources, lack of funding for genetics programs, access to genetic services, and cost of testing have been identified as key barriers in several contexts. Among breast cancer patients in the United States, out-of-pocket costs for testing combined with poor reimbursement has resulted in postponed testing and delayed treatment decisions. ${ }^{29}$ This factor may apply to the Canadian healthcare setting for tests that are not reimbursed, which varies by province. Among patients at risk for Huntington's disease, financial burden and opportunity costs of testing and time taken for appointments were identified as deterrents to genetic testing in British Columbia. ${ }^{30}$ Weldon and Hawkins ${ }^{29,30}$ also discuss difficulties with timing and inflexibility of the testing process as additional barriers that limit access and utility of genetic testing. Increasing costs of healthcare and limited perception of benefit are barriers to finding adequate funding models for genetic testing. ${ }^{31}$ This is exacerbated by a shortage of trained genetic professionals and a lack of efficient clinical care delivery models. ${ }^{31}$

Specific to kidney cancer, lack of knowledge about HRCC, the availability and benefits of testing, and treatment options for patients with positive testing further impair the delivery of genetic services. In a best-worst scaling experiment, physicians ranked the relative importance of factors that affect the decision to integrate genetic testing into their practice. The most important factor was type of test and its ability to prognosticate followed by physician training and availability of guidelines. These factors were considered more important than professional fees or the cost of genetic tests. $^{32}$ Several authors have identified a lack of knowledge and absence of guidelines as important barriers to establishing a coordinated system of referral and interpretation for genetic testing. ${ }^{12,31,33}$ We believe that, in addition to developing guidelines, appropriate knowledge translation activities are essential to improving provider knowledge about genetic testing.

Lastly, the current clinical practice and perception of genetic testing poses a systemic limitation to patient access. Respondents of our survey reported that lack of coordination between specialists and a need for more subspecialisation were important barriers in the care of patients with HRCC. Some respondents suggested that creating regional subspecialty centres for HRCC could improve access. This finding is similar to that of Weldon and colleagues, who identified poor timing of testing as a barrier to clinical utility. ${ }^{29}$ Testing can be time-consuming and test results were not necessarily available when treatment decisions needed to be made. ${ }^{29}$ Likewise Hamilton and colleagues performed an interview-based study of 5 specialities within the Veterans Administration facilities to assess the factors influencing organizational adoption and implementation of clinical genetic services. ${ }^{34}$ They identified multiple barriers similar to those identified in our study. In addition, they identified that genetic testing had limited compatibility with some disciplines of medicine, such as cardiology and primary care, but high compatibility with neurology and oncology. Overall, the sites with successful genetics services had knowledgeable clinicians interested in developing services and organizational-level facilitators to streamline the test-ordering process. On an organizational level, a different approach has been adopted in Alberta. Alberta has established a centralized process for requesting genetic testing that is not otherwise available. This approach seems to have resulted in improved consistency of testing and a costsavings, but may have resulted in reduced access for some patients. ${ }^{35}$ Out-of-province/country testing was identified as a barrier by respondents of our survey; perhaps a nationally centralized approach to ordering and conducting less common genetic testing is warranted. This would increase volumes, which may decrease costs. A nationally centralized approach could also assist in providing equal access across provinces in accord with The Canada Health Act. ${ }^{36}$

\section{Conclusion and practice implications}

Our study addresses an important gap regarding the use, provider knowledge, and perceptions of genetic testing for HRCC in Canada. We have identified a significant need to increase provider knowledge of genetic testing for HRCC. These findings support the development of practice guidelines and national strategies to improve the coordination of specialists and access to genetics services. We believe that HRCC provides an ideal context within which to develop these strategies.

\begin{abstract}
Acknowledgements: This survey was presented at the KCRNC Canadian Kidney Cancer Forum Genetics initiative in January 2014 for review by the following attendees who provided feedback for manuscript preparation: Melanie Care, Darrel Drachenberg, Andrew Evans, Craig Gedye, Ronald Grant, Jane Green, Eric Hyndman, Franny Jewett, Anthony Joshua, Anil Kapoor, Jean-Baptiste Lattouf, Michael Ohh, Stephen Pautler, Nadia Prigoda-Lee, Neil Reaume, Mark Robson, Alan So, Tracy Stockley, Philippe Violette, Andrew Weller. The Kidney Cancer Research Network of Canada is supported by grants from Kidney Cancer Canada, The Kidney Foundation of Canada, and a Network Catalyst Grant from the Canadian Institute of Health Research.
\end{abstract}

Competing interests: Dr. Violette, Dr. Graham, and Melanie Care declare no competing financial or personal interests. Dr. Kamel-Reid is a member of the Advisory Board for Novartis and has received grants from Novartis and BMS. Dr. Reaume is a member of the Advisory Boards for Pfizer, Sanofi-Aventis, Novartis, GlaxoSmithKline and Janssen. He is also participating in clinical trials with Pfizer, Exelis, GlaxoSmithKline, Boehringer Ingelheim, Astellas, Synta, BMS, Teva and Imclone. Dr. 
Violette et al.

Jewett is a member of the Advisory Board for Pfizer. He has also received grants from Novartis, GSK and Pfizer. Lastly, he has participated in clinical trials with Novartis, GSK and Pfizer. Ms. Basiuk has received grants from Pfizer and Novartis for presentations to nursing groups. Dr. Pautler has received unrestricted grants-in-aid from Paladin and Sanofi. He is also participating in an ongoing clinical trial with Astellas.

This paper has been peer-reviewed.

\section{References}

1. Siegel R, Naishadham D, Jemal A. Cancer statistics, 2013. CA Cancer J Clin 2013;63:11-30. http:// dx.doi.org/10.3322/caac.21166

2. Canadian Cancer Society. Canadian Cancer Statistics 2013. Toronto, Canadian Cancer Society; 2013.

3. Linehan WM, Srinivasan R, Schmidt LS. The genetic basis of kidney cancer: A metabolic disease. Nat Rev Urol 2010;7:277-85. http://dx.doi.org/10.1038/nrurol.2010.47

4. Chan-Smutko G. Genetic testing by cancer site: Urinary tract. Cancer J 2012;18:343-9. http://dx.doi. org/10.1097/PP0.0b013e31826246ac

5. Coleman JA. Familial and hereditary renal cancer syndromes. Urol Clin North Am 2008;35:563-72. http://dx.doi.org/10.1016/i.ucl.2008.07.014

6. Shuch B, Vourganti S, Ricketts CJ, et al. Defining early-onset kidney cancer: Implications for germline and somatic mutation testing and clinical management. J Clin Oncol 2014;32:431-7. http://dx.doi. org/10.1200/JC0.2013.50.8192

7. Coleman JA, Russo P. Hereditary and familial kidney cancer. Curr Opin Urol 2009;19:478-85. http:// dx.doi.org/10.1097/MOU.0b013e32832f0d40

8. Poulsen ML, Budtz-Jørgensen E, Bisgaard ML. Surveillance in von Hippel-Lindau disease (vHL). Clin Genet 2010;77:49-59. http://dx.doi.org/10.1111/i.1399-0004.2009.01281.x

9. FluidSurveys. www.fluidsurveys.com. Accessed November 10, 2014.

10. Neergaard MA, Olesen F, Andersen RS, et al. Qualitative description - the poor cousin of health research? BMC Med Res Methodol 2009;9:52. http://dx.doi.org/10.1186/1471-2288-9-52

11. Reaume MN, Graham GE, Tomiak E, et al. Canadian guideline on genetic screening for hereditary renal cell cancers. Can Urol Assoc J 2013;7:319-23. http://dx.doi.org/10.5489/cuaj.1496

12. Miller $F A$, Krueger $P$, Christensen RJ. Postal survey of physicians and laboratories: Practices and perceptions of molecular oncology testing. BMC Health Serv Res 2009;9:131. http://dx.doi.org/10.1186/14726963-9-131

13. Silversides A. The wide gap between genetic research and clinical needs. CMAJ 2007;176:315-6. http:// dx.doi.org/10.1503/cmaj.061726

14. Adair A, Hyde-Lay R, Einsiedel E, et al. Technology assessment and resource allocation for predictive genetic testing: A study of the perspectives of Canadian genetic health care providers. BMC Med Ethics 2009;10:6. http://dx.doi.org/10.1186/1472-6939-10-6

15. Ontario Genetics Secretariat. Genetic Testing, Services and Research, Contributing to the Future Health of Ontarians. White Paper; 2009

16. Genetic Resources Ontario. http://www.geneticresourcesontario.ca/. Accessed November 10, 2015.

17. Grada A, Weinbrecht K. Next-generation sequencing: methodology and application. J Invest Dermatol 2013;133:e11. http://dx.doi.org/10.1038/iid.2013.248
18. Miller FA, Carroll JC, Wilson BJ, et al.The primary care physician role in cancer genetics: A qualitative study of patient experience. Fam Pract 2010;27:563-9. http://dx.doi.org/10.1093/fampra/cmq035

19. Trepanier A, Ahrens $M$, Mckinnon W, et al. Genetic cancer risk assessment and counseling: Recommendations of the national society of genetic counselors. J Genet Couns 2004;13:83-114. http:// dx.doi.org/10.1023/B:JOGC.0000018821.48330.77

20. Zbar B, Glenn G, Merino M, et al. Familial renal carcinoma: Clinical evaluation, clinical subtypes and risk of renal carcinoma development. J Urol 2007;177:461-5. http://dx.doi.org/10.1016/i.juro.2006.09.037

21. Bradley S, Dumas N, Ludman $M$, et al. Hereditary renal cell carcinoma associated with von Hippel-Lindau disease: A description of a Nova Scotia cohort. Can Urol Assoc J 2009:3:32-6.

22. Verine J, Pluvinage $A$, Bousquet $G$, et al. Hereditary renal cancer syndromes: An update of a systematic review. Eur Urol 2010;58:701-10. http://dx.doi.org/10.1016/i.eururo.2010.08.031

23. Ricketts $\mathrm{CJ}$, Shuch B, Vocke CD, et al. Succinate dehydrogenase kidney cancer: An aggressive example of the Warburg effect in cancer. J Urol 2012;188:2063-71. http://dx.doi.org/10.1016/i.juro.2012.08.030

24. Linehan WM, Zbar B. Focus on kidney cancer. Cancer Cell 2004;6:223-8. http://dx.doi.org/10.1016/i. ccr.2004.09.006

25. Gerlinger $M$, Rowan AJ, Horswell $S$, et al. Intratumor heterogeneity and branched evolution revealed by multiregion sequencing. N Engl J Med 2012;366:883-92. http://dx.doi.org/10.1056/NEJMoal 113205

26. Longo DL. Tumor heterogeneity and personalized medicine. N Engl J Med 2012;366:956-7. http:// dx.doi.org/10.1056/NEJMe1200656

27. Shoij S, Nakano M, Sato H. The current status of tailor-made medicine with molecular biomarkers for patients with clear cell renal cell carcinoma. Clin Exp Metastasis 2014;31:111-34. http://dx.doi. org/10.1007/s10585-013-9612-7

28. Audenet $F$, Yates DR, Cancel-Tassin $G$, et al. Genetic pathways involved in carcinogenesis of clear cell renal cell carcinoma: Genomics towards personalized medicine. BJU Int 2012;109:1864-70. http:// dx.doi.org/10.1111/j.1464-410X.2011.10661.x

29. Weldon CB, Trosman JR, Gradishar WJ, et al. Barriers to the use of personalized medicine in breast cancer. J Oncol Pract 2012;8:e24-31. http://dx.doi.org/10.1200/JOP.2011.000448

30. Hawkins AK, Creighton S, Hayden MR. When access is an issue: Exploring barriers to predictive testing for Huntington disease in British Columbia, Canada. Eur J Hum Genet 2013;21:148-53. http://dx.doi. org/10.1038/eihg.2012.147

31. Deverka PA, Doksum T, Carlson RJ. Integrating molecular medicine into the US health-care system: Opportunities, barriers, and policy challenges. Clin Pharmacol Ther 2007;82:427-34. http://dx.doi. org $/ 10.1038 /$ si.clpt.6100319

32. Najafzadeh $M$, Lynd $L D$, Davis $J C$, et al. Barriers to integrating personalized medicine into clinical practice: A best-worst scaling choice experiment. Genet Med 2012;14:520-6. http://dx.doi.org/10.1038/ gim. 2011.26

33. Bonter $K$, Desiardins C, Currier N, et al. Personalised medicine in Canada: A survey of adoption and practice in oncology, cardiology and family medicine. BMJ Open 2011;1:e000110. http://dx.doi. org/10.1136/bmiopen-2011-000110

34. Hamilton $A B$, Oishi $S$, Yano $E M$, et al. Factors influencing organizational adoption and implementation of clinical genetic services. Genet Med 2014;16:238-45. http://dx.doi.org/10.1038/gim.2013.101

35. Lilley $M$, Christian S, Blumenschein P. A centralized approach to out-of-province genetic testing leads to cost savings: The Alberta experience. Clin Genet 2013;84:373-7. http://dx.doi.org/10.1111/cge.12077

36. Canada Health Act Revised Statutes of Canada (1985, c. C-6) Retrieved from the Department of Justice. http://laws-lois.justice.gc.ca/PDF/C-6.pdf. Accessed November 10, 2014

Correspondence: Dr. Stephen E. Pautler, Urology, St. Joseph's Hospital, 268 Grosvenor St., London, ON N6A 4V2; stephen.pauter@sihc.london.on.ca 